\title{
Análise da Produção Bibliográfica sobre Problem- Based Learning (PBL) em Quatro Periódicos Selecionados
}

\author{
Analysis of Bibliographic Production on Problem- \\ Based Learning (PBL) in Four Selected Journals
}

\author{
Ana Neiline Cavalcantel \\ Geison Vasconcelos Lira ${ }^{I I}$ \\ Pedro Gomes Cavalcante Neto ${ }^{I I}$ \\ Roberta Cavalcante Muniz Lira ${ }^{\text {II }}$
}

\section{PALAVRAS-CHAVE \\ - Aprendizagem Baseada em Problemas. \\ - Educação Médica. \\ - Revisão.}

REVISTA BRASILEIRA DE EDUCAÇĀO MÉDICA
${ }^{I}$ Instituto Superior de Teologia Aplicada, Sobral, $C E$, Brasil.

"Iniversidade Federal do Ceará, Sobral, CE, Brasil.

\begin{abstract}
RESUMO
Trata-se de uma revisão integrativa que analisa as características bibliométricas da produção científica empírica sobre PBL em periódicos de referência em educação médica no Brasil e no mundo, de 2005 a 2014, e recomenda direcionamentos para futuras pesquisas. Dos artigos do corpus, foram extraídos os seguintes dados: ano de publicação, cenário do estudo, objetivos do estudo, tipo de estudo, principais conclusões e diretrizes para pesquisas futuras assinaladas pelos autores. Os artigos publicados nos três periódicos internacionais tiveram por cenário escolas médicas de todos os continentes, sendo que seis estudos são multicêntricos. Os estudos nacionais se concentram nos estados das regiões Sul e Sudeste. Quanto aos objetivos dos estudos analisados, há predominância da busca da efetividade do $P B L$, ou seja, dos resultados, em termos de desempenho dos alunos, seja na formação ou na prática médica, comparando seu desempenho com o de alunos submetidos aos métodos tradicionais de ensino. Quanto à tendência da quantidade de artigos sobre PBL publicados nos últimos dez anos, observou-se uma significativa redução da quantidade de artigos publicados nos três periódicos internacionais de 2005 a 2010. No caso do periódico nacional, os dados sugerem que a produção científica sobre PBL no Brasil ainda é incipiente. No que se refere aos métodos de pesquisa utilizados nos estudos publicados nos quatro periódicos selecionados, houve predominância dos métodos quantitativos, com predomínio do survey $(n=26)$. As principais conclusões dos estudos seguem a mesma linha dos objetivos. Elas mostram os resultados positivos do PBL tanto na formação médica quanto em suas repercussão na prática profissional. Quanto às diretrizes para futuras pesquisas, há uma inclinação para a realização de mais estudos que investiguem a efetividade do PBL, assim como mais estudos comparativos. Conclui-se que a pesquisa sobre PBL é incipiente, sendo preciso realizar mais estudos que busquem responder a questões teórico-metodológicas e epistemológicas do método. Isto sem deixar de observar a qualidade das pesquisas que estão sendo conduzidas, pois, quanto mais qualidade tiverem, melhor irão subsidiar a tomada de decisões. Um dado promissor é que o PBL tem despertado o interesse de pesquisadores mundo afora. Isto mostra que o método, apesar de seus rigorosos passos, pode se adaptar a diversas culturas e contextos educacionais. Este estudo apresenta limitações, pois, para viabilizar a pesquisa, as buscas se reduziram a alguns periódicos e a um corte temporal de dez anos. Dessa forma, recomenda-se efetuar pesquisas que englobem mais periódicos e um espaço de tempo maior, para que se possa traçar um panorama mais completo das publicações sobre PBL.
\end{abstract}




\section{KEY WORDS}

- Problem-Based Learning.

- Medical Education.

- Review.
ABSTRACT

The present study is an integrative review. The aim was to analyze the bibliometric characteristics of empirical scientific literature on PBL in reference medical education journals in Brazil and in the world from 2005 to 2014, and recommend directions for future research. The following data were extracted: year of publication, study setting, study goals, study type, key findings and directions for future research indicated by the authors. The settings in the articles published in the three international journals were Medical Schools in all continents, where $n=6$ studies were multicentric. When we analyze the data for the distribution of national studies among states, we can see that they are concentrated in the South and Southeast of Brazil. As regards the objectives of the studies analyzed, we observed that a predominance of attempts to verify the effectiveness of PBL, that is, looking at results in terms of student performance, whether in training or medical practice, and by comparing such results to those obtained via traditional methods. As regards the trend in the number of articles on PBL published in the last 10 years, we observed a significant reduction in the number of articles published in the three international journals from 2005 to 2010. In the case of the national periodical, our data suggest that scientific literature on PBL in Brazil remains at an incipient stage. As regards the research methods used in the studies published in the four selected journals, we noted the predominance of quantitative methods, primarily through the use of surveys $(n=26)$. The main conclusions of the studies follow the same line of goals. They show the positive results of both PBL in medical education and their implications for professional practice. In terms of guidelines for future research, we see that there is an inclination to carry out further studies to investigate the effectiveness of PBL, as well as more comparative studies. In conclusion we can say that research on PBL is still in its infancy, we still need to move forward in further studies that seek to answer more theoretical and methodological issues and employ epistemological methods. The quality of research carried out also requires attention, because the higher the quality the better the research will support decision-making. A very promising finding is that PBL hás aroused the interest of researchers all over the world. This shows that the method, despite its rigorous steps, can adapt to different cultures and different educational contexts. However, we cannot fail to mention the limitations of this study. To enable the research, the search was reduced to a limited number of journals, and a timeframe of 10 years. Thus it is recommended that further research be conducted, encompassing more publications and a longer period of time, in order to draw a more complete picture of scientific literature on PBL.

Recebido: 27/01/2017

Aceito: 30/08/2017

\section{INTRODUÇÃO}

A Aprendizagem Baseada em Problemas ou PBL (sigla inglesa da expressão Problem-Based Learning), como uma metodologia ativa estimuladora do autoaprendizado e do pensamento crítico $^{1}$, é considerada uma das mais significativas inovações na educação médica nos últimos anos, tendo surgido como um movimento de reação aos currículos das escolas médicas sob a forte influência do modelo flexneriano, que privilegiava o modelo biomédico e o ensino centrado no hospital².

O PBL representa uma perspectiva de ensino-aprendizagem ancorada no construtivismo, ou seja, na (re)construção dos conhecimentos, cujo processo é centrado no estudante ${ }^{3}$. A aprendizagem, nessa perspectiva, resulta do processo de trabalho orientado para a compreensão e a resolução de um problema ${ }^{4}$. Ela traz uma mudança de concepção da relação professor-aluno, tendo o aluno como o sujeito ativo no processo de ensino-aprendizagem, o que diminui a distância entre esses dois sujeitos, muitas vezes considerados como polos dicotomizados e submetidos a uma rígida hierarquia. O professor passa a atuar como um orientador que auxilia o aluno a alcançar o objetivo de aprendizagem e não mais como único detentor do conhecimento ${ }^{4}$. A relação, assim, torna-se mais horizontalizada, e as relações de poder tendem a ser menos conflituosas e instituídas.

No Brasil, acompanhando uma tendência mundial em educação médica, as Diretrizes Curriculares Nacionais (DCN) 
para os Cursos de Graduação em Medicina, atualizadas em 2014, preconizam que os cursos devem utilizar metodologias ativas de aprendizagem na construção do conhecimento ${ }^{5}$, entre as quais se destaca o PBL.

O estudo dos currículos dos cursos de Medicina, com destaque para os currículos baseados em PBL, figura em primeiro lugar em pesquisas na área de educação médica ${ }^{6}$. De fato, os trabalhos que se ocuparam da temática dos currículos médicos sob a perspectiva da avaliação curricular enfocaram, predominantemente, os desfechos de currículos estruturados com base no $\mathrm{PBL}^{7-13}$ e nos componentes curriculares que seguem essa estratégia pedagógica ${ }^{14-17}$. Não obstante essas priorizações de enfoque, mesmo a análise crítica desses trabalhos suscitou importantes debates teórico-metodológicos, que fizeram avançar a compreensão da complexidade de se abordar o currículo médico como tema de pesquisa ${ }^{18-25}$.

Contudo, a questão metodológica não é o único desafio. Há também a questão do contexto de aplicabilidade. Alguns estudos indicam que, se o PBL não for executado como foi idealizado, ou seja, se todos os passos não forem seguidos, ou se não houver um constante investimento no desenvolvimento do corpo docente com vistas a uma mudança de habilidades e atitudes, o método pode acabar se distorcendo de tal maneira que professores e alunos continuariam a agir como se estivessem num ambiente de aprendizagem tradicional ${ }^{26}$.

A Society of Directors of Research in Medical Education criou uma agenda para revisões sistemáticas na área de educação médica, na qual os três temas prioritários eram desenho curricular, avaliação e resultados, tendo em vista que esses são os problemas mais recorrentes no que se refere à qualidade de evidências na literatura ${ }^{27}$. Nesse contexto, o PBL permanece um tema relevante de investigação.

É possível supor que essas diretrizes de pesquisa tenham se materializado nos últimos anos, resultando em publicações com potencial para fornecer evidências que embasem decisões de mudanças curriculares que privilegiem metodologias ativas de aprendizagem, com destaque para o PBL, particularmente no Brasil, onde as DCN sinalizam nessa direção.

Assim, realizamos uma pesquisa bibliográfica com os objetivos de analisar as características bibliométricas da produção científica empírica sobre PBL em periódicos de referência em educação médica no Brasil e no mundo, de 2005 a 2014, e recomendar direcionamentos para futuras pesquisas.

\section{MATERIAL E MÉTODOS}

\section{Método}

Foi realizada uma revisão integrativa, método de pesquisa que tem a finalidade de reunir e sintetizar resultados de pesquisas sobre determinado tema ou questão, de maneira sistemática e ordenada, contribuindo para aprofundar o conhecimento do tema investigado ${ }^{28}$. Para a construção da revisão integrativa, é preciso percorrer seis etapas: identificação do tema e seleção da hipótese ou questão de pesquisa; estabelecimento de critérios para inclusão e exclusão de estudos; definição dos estudos selecionados e pré-selecionados; categorização dos estudos; análise e interpretação dos resultados apresentação da revisão/síntese do conhecimento ${ }^{29}$.

\section{Critérios de inclusão}

Foram aplicados os seguintes critérios de inclusão: artigos originais que tinham como tema central o PBL, de pesquisa empírica ou de relatos de experiência, publicados nos últimos dez anos, em três periódicos internacionais de referência em educação médica - Medical Teacher, Academic Medicine e Medical Education - e em um periódico nacional de referência no mesmo campo - Revista Brasileira de Educação Médica.

\section{Estratégia e período de busca}

A estratégia de busca foi realizada em três etapas. Inicialmente, fez-se uma pesquisa na Revista Brasileira de Educação Médica, disponível na base de dados eletrônica SciELO, utilizando-se como orientadores de busca os seguintes descritores: Problem-Based Learning, Problem-Based Education, Problem-Based in Learning, Problem-Based Learning, Problem-Based Teaching, Problem-Based Teaching and Learning, Problem-Posing Education, Aprendizagem Baseada em Problema, Aprendizagem Baseada em Problemas, Aprendizagem Baseada em Problemas Ensino e Pesquisa. Tais descritores foram obtidos entre os Descritores em Ciências da Saúde (DeCS), para identificar produções bibliográficas que tenham como tema o PBL. Como, numa exploração preliminar, identificou-se que na SciELO constavam apenas os números a partir de dezembro de 2006, empreendeu-se uma busca na base de dados da Bireme com os mesmos descritores citados acima para localizar os números de janeiro a dezembro de 2005 e de 2006. Em seguida, fez-se uma pesquisa na base de dados Pubmed para identificar publicações nas revistas Medical Teacher, Academic Medicine e Medical Education, utilizando-se como orientadores de buscas os seguintes termos, obtidos entre os Mesh Terms: Undergraduate Medical Education, Medical Schools, Medical Education, Medical Students, Problem-Based Learning, Problem-Based Curriculum, Experiential Learning, Active Learning, Problem Solving. A busca foi feita nos meses de junho e julho de 2015 pela autora deste trabalho e auditada por outros dois pesquisadores independentes, para assegurar sua qualidade.

Como produto da busca inicial, foram encontrados 842 artigos nos três periódicos internacionais e 33 no periódico 
nacional. O processo de seleção dos artigos para inclusão no corpus de análise deu-se da seguinte forma:

- Primeiro passo: foram lidos títulos e resumos dos artigos encontrados para que fossem excluídos aqueles que não tinham relação com a temática do PBL. Neste passo, foram excluídos 685 artigos, 682 dos periódicos internacionais e três do periódico nacional;

- Segundo passo: foram lidos os artigos na íntegra para que fossem incluídos no corpus apenas aqueles de pesquisa empírica ou de relato de experiência. Neste passo, foram excluídos 117 artigos dos periódicos internacionais e 21 do periódico nacional.

No final do processo, restaram 52 artigos para extração dos dados, que compuseram o corpus da revisão, sendo 40 obtidos dos periódicos internacionais e 12 do periódico nacional. Uma síntese do processo de seleção é apresentada na Figura 1.

\section{Figura 1}

Descrição do método de seleção da revisão interativa sobre Problem-Based Learning - junho e julho de 2015

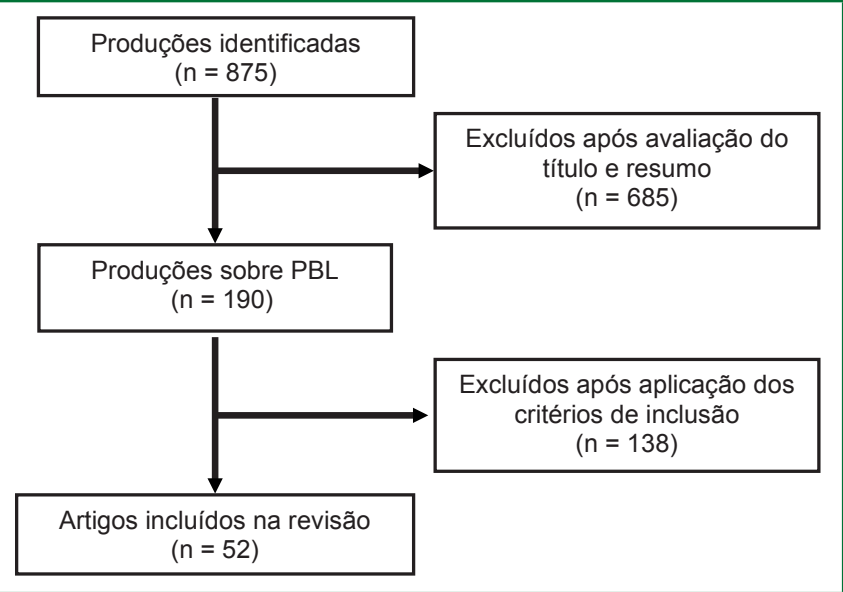

\section{Extração dos dados}

Dos artigos do corpus, foram extraídos os seguintes dados: ano de publicação, cenário do estudo, objetivos do estudo, tipo de estudo, principais conclusões e diretrizes para pesquisas futuras. Uma síntese dos dados extraídos de cada artigo foi elaborada, utilizando-se uma tabela padronizada para cada periódico, a qual foi utilizada para análise.

\section{RESULTADOS}

Quanto ao cenário de estudo, dos 12 estudos desenvolvidos no Brasil, 11 foram realizados em instituições localizadas nas regiões Sul e Sudeste e um na Região Centro-Oeste do País
(Tabela 1). Todos os estudos foram realizados em instituições que adotam o método PBL.

TABela 1

Distribuição das publicações por estados

que sediavam as escolas médicas que foram

cenários dos estudos sobre PBL, na Revista

Brasileira de Educação Médica: 2005-2014

Cenário

Número de Publicações

Brasília

1

São Paulo

Rio de Janeiro

1

Minas Gerais

3

Paraná

Porto Alegre

As publicações internacionais referiram-se a pesquisas realizadas em todos os continentes, sendo 31 na Europa, 10 na América e na Ásia, 2 na África e 2 na Oceania (Tabela 2). Dos 40 estudos, 4 foram multicêntricos, realizados em escolas médicas da Europa, da América e da Ásia.

\section{TABELA 2}

Distribuição das publicações pelos continentes e

países que sediavam as escolas médicas que foram

cenários dos estudos sobre PBL, em Medical Teacher,

Academic Medicine e Medical Education: 2005-2014

Cenário

Número de Publicações

Europa

31

América

África

11

Ásia

2

Oceania

10

2

Quanto à tendência da quantidade de artigos publicados nos últimos dez anos, o Gráfico 1 apresenta a distribuição da publicação dos artigos nos três periódicos internacionais. Depreende-se do gráfico que a publicação de artigos sobre PBL nesse período predominou nas revistas Medical Teacher e Medical Education, sendo que de 2008 a 2010 os artigos foram publicados exclusivamente na Medical Education, e, nos anos de 2011, 2013 e 2014, foram publicados exclusivamente na $\mathrm{Me}$ dical Teacher. 


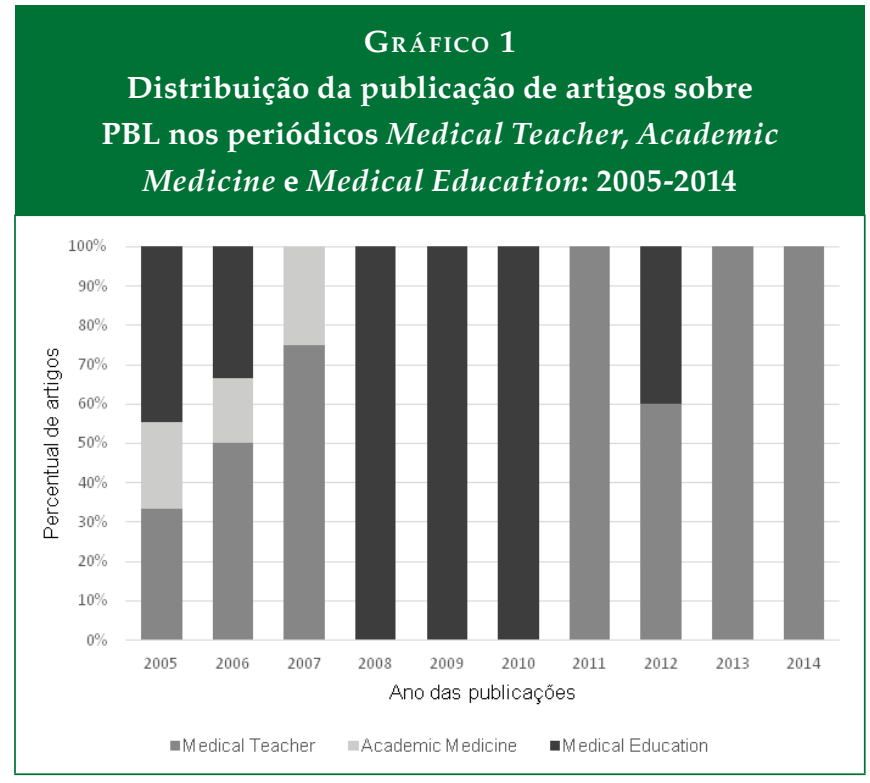

O Gráfico 2 mostra uma tendência de queda no número de publicações de artigos nos três periódicos internacionais, alcançando seu mínimo em 2010. No periódico nacional, por seu turno, percebe-se uma tendência discreta de aumento na quantidade de artigos publicados, aproximando-se da quantidade publicada nos três periódicos internacionais, mais pela queda na quantidade das publicações destes últimos do que por um aumento expressivo da quantidade de publicações no periódico nacional.

\section{GrÁfico 2}

Tendência do número de artigos sobre PBL publicados nos três periódicos internacionais

(Medical Teacher, Academic Medicine e Medical Education) e no periódico nacional (Revista Brasileira de Educação Médica): 2005-2014

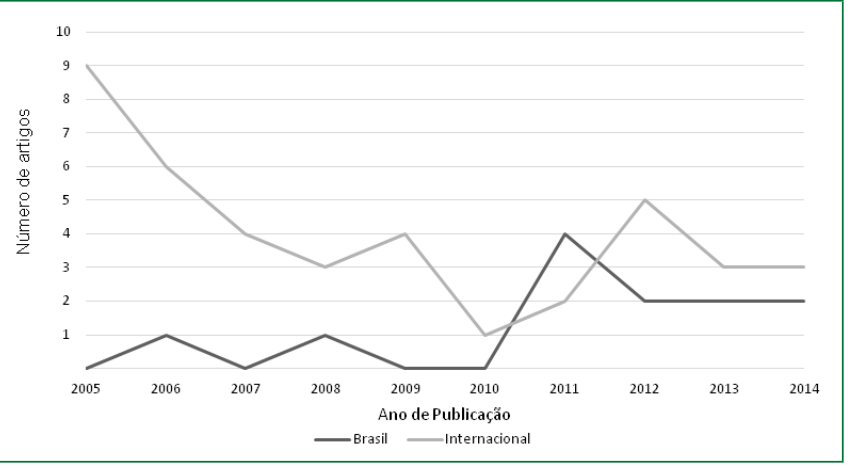

Os principais objetivos dos estudos foram bem variados, como apresentado na Tabela 3. Os mais frequentes referiram-se à demonstração da efetividade do PBL, seja aferindo seus impactos na formação dos estudantes (12), seja comparando o currículo baseado em PBL com outros modelos curriculares (11).

\begin{tabular}{|lc|} 
TABELA 3 & \\
Categorização dos principais objetivos dos & \\
estudos sobre PBL publicados em Medical Teacher, \\
Academic Medicine, Medical Education e Revista \\
Brasileira de Educação Médica: & 2005-2014 \\
\hline Objetivos & Frequência \\
\hline Impactos do PBL na formação médica & 12 \\
\hline Comparação do PBL com outros modelos de currículo & 11 \\
\hline Variações no modo de usar o método PBL & 4 \\
\hline Concepções dos docentes sobre o PBL & 3 \\
\hline Desempenho dos estudantes no método PBL & 3 \\
\hline Outros & 18
\end{tabular}

Com respeito aos métodos utilizados nos 52 artigos selecionados, os mais utilizados, como se pode observar na Tabela 4, foram: survey (26), estudos de caso (6), estudos de intervenção (6) e estudos exploratórios (5).

\begin{tabular}{|c|c|}
\hline $\begin{array}{l}\text { Métodos utilizados nos } \\
\text { Medical Teacher, Acader } \\
\quad \text { e Revista Brasileira }\end{array}$ & $\begin{array}{l}\text { PBL publicados em } \\
\text { Medical Education } \\
\text { édica: 2005-2014 }\end{array}$ \\
\hline Métodos dos estudos & Frequência \\
\hline Survey & 26 \\
\hline Estudo de intervenção & 6 \\
\hline Estudo de caso & 6 \\
\hline Estudo exploratório & 5 \\
\hline Estudo de avaliação & 2 \\
\hline Ensaio clínico randomizado & 1 \\
\hline Outros & 6 \\
\hline
\end{tabular}

Sobre as principais conclusões dos 52 estudos selecionados, 13 concluíram que o PBL tem repercussões positivas na formação médica (Tabela 5). Além da influência na formação, cinco estudos também concluíram que o PBL tem repercussões positivas na prática médica. Outro dado que chama atenção é a melhoria da participação docente por meio do PBL na formação médica e a ajuda do PBL na aquisição de habilidades e atitudes, ambos com cinco estudos cada.

$17 \mid$\begin{tabular}{l|l} 
REVISTA BRASILEIRA DE EDUCAÇÃO MÉDICA \\
42 (1):10 - $4 ; 2018$
\end{tabular}

$1 7 \longdiv { 4 2 ( 1 ) : 1 0 - 2 4 ; 2 0 1 8 }$ 


\begin{tabular}{|c|c|}
\hline $\begin{array}{c}\text { TABELA } 5 \\
\text { Categorização das principais conclusões } \\
\text { estudos sobre PBL publicados em Medical T } \\
\text { Academic Medicine, Medical Education e } R \\
\text { Brasileira de Educação Médica: } 2005-20\end{array}$ & $\begin{array}{l}\text { dos } \\
\text { eacher, } \\
\text { evista } \\
14\end{array}$ \\
\hline Conclusões & Frequência \\
\hline O PBL tem repercussões positivas na formação médica & 13 \\
\hline $\begin{array}{l}\text { O PBL melhora a participação docente na formação } \\
\text { dos alunos }\end{array}$ & 6 \\
\hline O PBL tem repercussões positivas na prática médica & 5 \\
\hline $\begin{array}{l}\text { O uso do PBL ajuda na aquisição de habilidades e } \\
\text { atitudes }\end{array}$ & 5 \\
\hline O PBL favorece a integração entre teoria e prática & 4 \\
\hline $\begin{array}{l}\text { Deve haver maior preocupação com a educação } \\
\text { permanente dos docentes }\end{array}$ & 4 \\
\hline $\begin{array}{l}\text { Case method é mais adequado do que PBL para as fases } \\
\text { posteriores da formação médica }\end{array}$ & 4 \\
\hline Outros & 11 \\
\hline
\end{tabular}

No que diz respeito às diretrizes para futuras pesquisas, dos 52 artigos selecionados, 26 as indicaram (Tabela 6). As que mais apareceram foram a necessidade de novos estudos que investiguem a efetividade do PBL, tanto para a formação como para a prática médica (12). Outra diretriz que teve destaque foi a necessidade de mais estudos comparativos entre o PBL e outros métodos de ensino. Ou seja, dos 52 artigos selecionados, 17 assinalaram a necessidade de continuar investigando a efetividade do método. Um dado a assinalar é que três artigos sugeriram a necessidade de realizar estudos multicêntricos.

\section{TABELA 6}

Categorização das diretrizes para futuras pesquisas indicadas pelos estudos sobre PBL publicados em

Medical Teacher, Academic Medicine, Medical Education e Revista Brasileira de Educação Médica: 2005-2014

Diretrizes

Frequência

Realizar mais estudos que investiguem a efetividade da aprendizagem do PBL na prática médica

Realizar estudos que investiguem mais a efetividade da aprendizagem do PBL na formação médica

Realizar estudos comparativos com outros métodos de ensino

Outros

\section{DISCUSSÃO}

Os artigos publicados nos três periódicos internacionais selecionados tiveram por cenário escolas médicas de todos os continentes, sendo que quatro estudos eram multicêntricos. Esses dados mostram a ubiquidade da preocupação em tomar o PBL como objeto de pesquisa, acompanhando, certamente, a difusão internacional do método, no esteio dos movimentos recentes de mudança em educação médica que têm sido reportados internacionalmente em países/regiões diversos, tais como França $^{30}$, Índia ${ }^{31}$, China ${ }^{32}$, Japão ${ }^{33}$, Austrália ${ }^{34}$, Sudeste europeu $^{35}$, América Latina ${ }^{36,37}$ e Sudeste asiático ${ }^{38}$.

Por outro lado, quando analisamos os dados referentes à distribuição dos estudos realizados no Brasil entre seus diferentes estados, vemos que se concentram nas regiões Sul e Sudeste. Corroborando esse achado, uma revisão realizada em $2010^{39}$, que mapeou a produção científica em educação médica publicada na mesma Revista Brasileira de Educação Médica, de 2006 a 2010, observou que tal produção se concentra nessas mesmas regiões. Com efeito, podemos sugerir como hipóteses: 1) localização, nessas regiões, das instituições pioneiras no método PBL no Brasil; 2) maior tradição em pesquisa em instituições de ensino nessas regiões; 3) maior acesso dos pesquisadores dessas regiões a fomento à pesquisa.

Neste ponto é importante dizer que todos os estudos foram realizados em instituições que adotam o método PBL. Isto traz um possível problema de conflito de interesses nos estudos, decorrente do fato de que quem estuda PBL talvez seja majoritariamente praticante de PBL.

No tocante aos objetivos dos estudos analisados, observamos que há predominância na busca da efetividade do PBL em termos de desempenho - ou na formação ou na prática médica - dos alunos submetidos a esse método, e em termos de comparação entre o desempenho obtido por meio do PBL com aquele resultante de métodos convencionais de ensino. Uma hipótese para essa prevalência seria a necessidade de afirmar a efetividade do PBL perante o método tradicional. No entanto, não tem recebido a devida atenção dos pesquisadores a explicação das bases teóricas e metodológicas do método, nem a relação do PBL com a melhoria da saúde dos pacientes.

Nas últimas décadas, alguns autores passaram a se preocupar em entender as características de um currículo PBL, seus resultados e seu impacto como uma nova proposta para a educação médica. A literatura sobre o PBL tem se caracterizado mais pela descrição de experiências do que por uma análise crítica do paradigma da solução de problemas e dos modelos curriculares que o utilizam ${ }^{40}$. Outro estudo ${ }^{41}$ também corrobora essa hipótese ao sustentar que a maioria das pesquisas examina os processos locais e se baseia na satisfação do aluno e na aquisição de conhecimento de curto prazo para medir a efetividade educacional. Confrontando a tese dos relatos de experiência e dos estudos locais com nossos resultados, vemos que ela não se confirma nos quatro periódicos selecionados,

REVISTA BRASILEIRA DE EDUCAÇ̃̃O MÉDICA

$18{ }_{42(1): 10-24 ; 2018}$ 
visto que apenas seis artigos advêm de estudos de caso e que tem havido estudos multicêntricos ${ }^{54-57}$.

Quanto à tendência da quantidade de artigos sobre PBL publicados nos últimos dez anos nos quatro periódicos selecionados, observamos uma significativa redução nos três periódicos internacionais de 2005 a 2010. Por que houve essa queda? Uma hipótese a investigar é a possibilidade de que os pesquisadores estejam enfrentando dificuldades para demonstrar a efetividade e superioridade do PBL em relação aos métodos convencionais de ensino. Outra hipótese é a de que o PBL começou a ser estudado há décadas na Europa e América do Norte, e, dessa forma, é natural que o tempo tenha levado a um certo esgotamento das publicações na área.

Havendo essa dificuldade, seria necessário elucidar por que razões as publicações subsistiriam. Em primeiro lugar, é necessário considerar que grande parte da investigação é conduzida num cenário aplicado, prático, onde os educadores não têm a pesquisa educacional como prioridade. Em segundo lugar, a educação médica ocorre num contexto dinâmico, onde as demandas são resolvidas com ou sem pesquisa para direcionar e avaliar as decisões. Há pouca oportunidade para retardar ou parar a ação para projetar um estudo ideal ${ }^{42}$. Outro ponto a considerar diz respeito às questões de metodologia de investigação em educação médica, a demandar a proposição de novos métodos de pesquisa para investigação dos fenômenos educacionais em educação médica ${ }^{43}$, conforme discutiremos adiante.

Outra hipótese, mais radical, para essa queda na quantidade de artigos publicados seria que os estudiosos estariam chegando à conclusão de que não há superioridade do método PBL em relação aos métodos convencionais. Neste ponto, vale retomar a discussão entre autores que, no início dos anos 2000, polemizaram sobre a efetividade do $\mathrm{PBL}^{18,19}$.

Colliver ${ }^{18}$, após revisão de artigos sobre o PBL publicados de 1992 a 1998, conclui que não há evidência de que esse método melhore a base de conhecimento e o desempenho clínico, pelo menos não com a magnitude que seria desejada devido aos recursos e insumos necessários à organização de um currículo baseado em PBL. O autor atribui parte da responsabilidade à estruturação teórico-metodológica da psicologia cognitiva, alegando sua imprecisão. Esse estudioso critica os dois fatores que sustentam o PBL: o contexto e a ativação do conhecimento. Sobre o contexto, refere que tanto o ensino tradicional quanto o ensino em PBL não ocorrem no contexto da prática clínica e que, mesmo que os problemas simulados procurem se aproximar dos cenários reais de prática, não existe garantia de que os estudantes serão bem-sucedidos na prática clínica. Sobre a maior ativação das redes de conhecimentos no PBL, ele considera que essa afirmação é vaga, faltando clareza quanto a seu significado e sua verdadeira função. Além disso, não estão claros para ele os fatores que as ativam e se estímulos diferentes ativam as redes com intensidades e quantidades diferentes. Ele conclui concordando em que o "PBL pode prover um enfoque mais desafiante, motivador e agradável para a Educação Médica, mas sua efetividade educacional, comparada aos métodos convencionais, ainda deve ser demonstrada"18 (p. 266).

Norman e Schmidt ${ }^{19}$, por outro lado, confrontam a tese apresentada acima em dois pontos fundamentais: defendem que só se deve implantar o PBL se não houver custo excessivo; e, em relação às premissas teóricas, não consideram a psicologia cognitiva fraca, mas salientam que é preciso desenvolver programas de pesquisa sistemática, englobando todos os aspectos da construção e da avaliação da teoria da aprendizagem subjacente ao método, em projetos experimentais rigorosos e em cenários artificiais altamente controlados. Ou seja, eles sustentam que não se deve abandonar o método, mas que se empreendam mais pesquisas buscando a sua efetividade, com o devido rigor metodológico.

Nossos dados indicam que as linhas de pesquisa apontadas não se concretizaram nos artigos dos quatro periódicos selecionados, sugerindo que estão em aberto importantes questões de pesquisa que precisam ser respondidas para refutar a tese de Colliver $^{18}$. A afirmação deste de que a efetividade do PBL como método ainda deve ser comprovada permanece válida.

Dessa forma, e nossos dados o sugerem, ainda estão por ser obtidas, na literatura especializada, evidências indiscutíveis de que o PBL leve a melhores resultados de aprendizagem, resolução de problemas e transferência de conhecimentos para a prática profissional do que as formas convencionais de ensino. E o preocupante é que, ainda conforme nossos dados, essa busca pode estar arrefecendo entre os estudiosos do assunto.

No caso do periódico nacional, nossos dados sugerem que a produção científica sobre PBL no Brasil ainda é incipiente. De fato, a citada revisão feita por Hamamoto-Filho et al..$^{39}$ no periódico nacional selecionado para este estudo, a Revista Brasileira de Educação Médica, indicou que o descritor "Aprendizagem Baseada em Problemas" foi um dos menos frequentes (em dez artigos). Tal fato pode-se dever às seguintes hipóteses: não disseminação do PBL como método de ensino e como estratégia de reorganização curricular no Brasil, falta de tradição de pesquisa em educação médica no Brasil e, em consequência, pouco acesso a financiamento para pesquisa no âmbito desse campo.

No que se refere aos métodos de pesquisa utilizados nos estudos publicados nos quatro periódicos selecionados, ob- 
servamos a predominância dos métodos quantitativos, com predomínio do survey (26). Esse dado é corroborado em outro estudo $^{42}$ que indica que a pesquisa no campo da educação médica tem sido dominada por estudos quantitativos em detrimento dos qualitativos, ainda que estes estejam aumentando. Essa tendência, contudo, não foi observada em nossos dados.

Há, de fato, uma tendência na pesquisa em educação médica de obter evidências de eficácia dos vários métodos de ensino, como, de resto, se observa no âmbito maior da Educação $^{44}$. Essa busca por melhores evidências na educação médica guiou a criação da Best Evidence Medical Education (Beme) Collaboration, com o intuito de criar um fluxo de revisões sistemáticas sobre o campo. Essas revisões orientariam a prática educacional e o trabalho de pesquisadores e avaliadores, pois conseguiria apontar lacunas (e, assim, incentivar a investigação em áreas para melhor entender o que e como se faz), assim como melhoraria a teoria com base na qual os programas são construídos ${ }^{26}$.

No âmbito da educação médica, sob influência da própria pesquisa clínica, alguns autores sugerem que devem ser privilegiados os métodos epidemiológicos para sustentar evidências de boa qualidade. Com efeito, na pesquisa clínica, o método, por excelência, é o experimento, enquanto a técnica de pesquisa considerada padrão ouro é o ensaio clínico randomizado cego, principalmente quando se pretende estabelecer relações putativas de causa e efeito ${ }^{45,46,47}$. Ao longo do desenvolvimento do campo da pesquisa em educação médica, essa técnica, por analogia com o campo da pesquisa clínica, passa a ser entendida, também, por pesquisadores e órgãos governamentais e fundações financiadoras, como aquela que assegura uma pesquisa rigorosa e de boa qualidade ${ }^{19,48}$.

Um artigo de comunicação especial ${ }^{49}$ chega a propor uma nova disciplina, a "Epidemiologia Educacional", que corresponderia à aplicação, ao continuum do processo de formação médica, dos métodos científicos existentes na pesquisa clínica - os desenhos observacionais (estudos transversais, de coorte e de caso-controle) e os desenhos experimentais randomizados (ensaios clínicos randomizados e desenhos cruzados randomizados). Estes últimos, no âmbito da Epidemiologia, têm a função de conduzir uma análise sem vieses de fatores associados com dado desfecho em dois ou mais grupos. Todos eles podem tanto gerar hipóteses quanto testá-las. Segundo os autores do artigo, os princípios que sustentam esses desenhos podem ser aplicados ao estudo dos desfechos educacionais, uma vez que, em educação médica, os estudantes constituem "populações" que fazem escolhas independentes sobre eventos que potencialmente influenciam competências eventuais que terão como médicos. Ademais, muitos desfechos de curto e longo prazos podem ser identificados, e, com recurso aos desenhos epidemiológicos listados acima, podem-se produzir evidências de que tais desfechos se associam a intervenções educativas entabuladas ou são causados por elas. Os autores concluem que a "Epidemiologia Educacional" pode gerar uma base de pesquisa poderosa para sustentar estudos desenhados para determinar a efetividade das intervenções educativas. Isso seria conseguido buscando-se a objetividade como elemento nuclear da investigação científica, que deve ser aplicada tanto à pesquisa educacional quanto à pesquisa biomédica. Segundo eles, "o poder desta objetividade e sua aplicação a como, na realidade, a prática médica é, e a como ela deveria ser ensinada promoveriam tanto seu êxito quanto o reconhecimento que lhe é devido" ${ }^{49}$ (p. 1049).

Tal perspectiva, fundada na objetividade, que é pressuposto da ciência normal biomédica, propõe uma "educação médica baseada em evidências", capaz de beneficiar a todos, incluindo educadores, estudantes e, principalmente, os pacientes, por meio da minimização de custos, da redução de erros médicos e da má prática profissional, e da maximização da qualidade do cuidado ${ }^{26}$. Nesse sentido, a despeito das louváveis intenções dessa perspectiva, outro autor ${ }^{43}$ sustenta que a "Epidemiologia Educacional" representa uma contaminação indevida dos fenômenos educacionais/educativos em educação médica pela orientação lógico-semântica da Biomedicina. Tais fenômenos são, assim, tomados por analogia aos fenômenos mórbidos, de que é objeto a ciência biomédica. Os fenômenos educacionais/educativos não são tomados em sua especificidade e complexidade, e, portanto, não são apreendidos por meio de procedimentos científicos que lhes são próprios e que tomam parte das ciências da Educação.

Mas, mesmo se adotarmos a perspectiva da "Epidemiologia Educacional", considerando o ensaio clínico randomizado cego como padrão ouro para demonstrar a efetividade do PBL, produzindo, assim, evidência de alta qualidade, nossos dados indicam que se está longe dessa diretriz, uma vez que apenas um estudo utilizou esse método de pesquisa ${ }^{50}$.

Contudo, é importante frisar que um ponto a considerar é que deve haver uma preocupação genuína com o rigor dos métodos de pesquisa, sejam eles quantitativos ou qualitativos, ainda um desafio no âmbito da pesquisa em educação médica. A falta de rigor nas pesquisas nesse campo pode ser devida a três fatores: condições de trabalho dos investigadores, restrições orçamentárias em financiamento para investigação em educação médica e a concepção do que seja pesquisa rigorosa no campo da Medicina. Para melhorar esse panorama, seria necessário intensificar a colaboração entre pesquisadores e médicos, e incentivar a diversificação de métodos, aborda- 
gens e objetos de pesquisa da investigação no campo ${ }^{51}$. A aplicação adequada do desenho do estudo e o desenvolvimento de resultados clinicamente significativos têm o potencial para tornar a pesquisa em educação médica mais relevante para as necessidades de saúde da população ${ }^{41}$.

As principais conclusões dos estudos seguem a mesma linha dos seus objetivos. Elas mostram os resultados positivos do PBL tanto na formação médica quanto em suas repercussões para a prática profissional. Alguns estudos ${ }^{52}$ apresentam conclusões semelhantes, pois mostram que os alunos submetidos ao PBL apresentam resultados superiores em diversas competências. Outro dado que chama atenção é a participação docente no método PBL. Vimos, na Tabela 4, que o PBL melhora a participação docente na formação dos alunos. Em revisão realizada em $2009^{53}$, encontramos um achado que corrobora essa conclusão. Segundo seus autores, o PBL constitui uma abordagem de ensino alternativa e, ao mesmo tempo, inovadora e é capaz de promover rupturas com o modelo convencional de ensino, estimulando a participação docente e a reorganização da relação entre teoria e prática.

No entanto, se olharmos esses dados pela lupa da "Epidemiologia Educacional", essas conclusões não estão embasadas em pesquisas com desenho metodológico de boa qualidade. Dessa forma, seguindo essa perspectiva, são necessárias novas pesquisas mais rigorosas quanto ao método, para se afirmar a real superioridade do PBL frente aos métodos convencionais. Contudo, como já assinalado, é necessária uma definição paradigmática sobre os métodos apropriados à pesquisa em educação médica, que deva se basear também nos referenciais teórico-metodológicos no campo mais amplo da pesquisa educacional ${ }^{43}$.

Quanto às diretrizes para futuras pesquisas expostas nos artigos analisados, há uma inclinação para a realização de mais estudos que investiguem a efetividade do PBL, assim como mais estudos comparativos. Isso mostra que, até aqui, as evidências produzidas são insuficientes para demonstrar a eficácia do método e sua superioridade relativamente aos métodos convencionais. A realização desses estudos pode trazer mais evidências para embasar a tomada de decisões das instituições de ensino sobre qual método é mais adequado às suas respectivas realidades. Alguns autores ${ }^{26}$ sustentam que existe a necessidade de mais pesquisa em educação médica para continuar a abordar questões-chave no campo. A própria natureza do ser profissional no contexto social de hoje exige que os educadores médicos forneçam provas da eficácia, da efetividade e da eficiência de seus programas.

\section{CONSIDERAÇÕES FINAIS}

Como principais conclusões deste trabalho, temos:

- Quanto à tendência do número de artigos sobre PBL publicados nos últimos dez anos nos três periódicos internacionais e no periódico nacional selecionados, nossos dados sugerem que a pesquisa sobre PBL ainda é incipiente e que ainda precisamos avançar na realização de mais estudos que busquem responder a questões mais teórico-metodológicas e epistemológicas do método;

- Quanto à distribuição dos artigos publicados nos três periódicos internacionais selecionados, o PBL tem despertado o interesse de pesquisadores em vários continentes. Portanto, apesar de seus passos rigorosamente sistematizados, o método pode se adaptar a diversas culturas e contatos educacionais diferentes, aspecto corroborado pela abrangência dos cenários onde os estudos foram realizados;

- Quanto aos principais objetivos dos estudos, o foco é no resultado/desempenho dos alunos submetidos ao PBL, tanto durante o período de formação como na prática médica, e na comparação desses resultados com aqueles obtidos com os métodos convencionais;

- No que se refere ao tipo de método empregado, sob a luz da "Epidemiologia Educacional", os mesmos não seriam os ideais para prover dados confiáveis. Essa questão da qualidade não pode deixar de ser observada, pois, quanto mais qualidade tiverem, melhor irão subsidiar a tomada de decisões;

- Quanto às principais conclusões dos estudos, existe uma superioridade de resultados dos alunos submetidos ao PBL, principalmente nas competências educacionais relacionadas ao social. Além desses resultados promissores na formação médica, o PBL contribui para a interação entre docentes e alunos, visto que melhora a participação docente na formação dos estudantes;

- No que se refere às diretrizes para novas pesquisas apontadas nos artigos, embora existam vários estudos que buscaram mostrar a efetividade do PBL na formação médica, ainda são necessários estudos que investiguem mais a fundo essa efetividade, assim como também mais estudos comparativos para mostrar a superioridade do PBL perante os métodos convencionais de ensino;

- Além das diretrizes apontadas nos artigos inclusos na revisão, nossos resultados mostram que são necessários novos estudos que mostrem os motivos da queda da produção internacional e que investiguem as bases teóricas e metodológicas do método e a relação do PBL com a melhoria da saúde dos pacientes. 
Embora o presente estudo tenha descrito muitas características bibliométricas das publicações sobre PBL nos quatro periódicos selecionados, não podemos deixar de citar suas limitações, visto que tivemos que reduzir as buscas a quatro periódicos (três internacionais e um nacional), além de fazer um corte temporal de dez anos. Dessa forma, recomenda-se realizar pesquisas que englobem mais periódicos ou mesmo incluir a chamada literatura cinzenta, abrangendo um espaço de tempo maior. Assim, será possível traçar um panorama mais completo das publicações sobre PBL.

Os resultados desta pesquisa poderão subsidiar a tomada de decisão, tanto de gestores quanto de docentes, de implementar o PBL em novos currículos ou aplicar melhorias nos currículos de PBL existentes.

\section{REFERÊNCIAS}

1. Caron CR. Aprendizagem problematizada no ensino médico na perspectiva do construtivismo piagetiano. Curitiba; 2004. Mestrado [Dissertação] - Universidade Federal do Paraná.

2. Schmidt H, et.al. Influence of Tutors subject-Matter Expertise on Student Effrort and Achievment in Problem-Based Learning. AcadMed 1993;62;784-791.

3. Moraes MAA, Manzini EJ. Concepções sobre a Aprendizagem Baseada em Problemas: um Estudo de Caso na Famema. RevBrasEducMéd 2006;30(3).

4. Mamede S, Penaforte JC. (Orgs.). Aprendizagem baseada em problemas: anatomia de uma nova abordagem educacional. Fortaleza: Hucitec, 2001.

5. Brasil. Ministério da Educação. Conselho Nacional de Educação. Câmara de Educação Superior. Resolução No 3, de 20 de junho de 2014.Institui diretrizes curriculares nacionais do curso de graduação em Medicina. Brasília, 20 de junho de 2014.

6. Regher G. Trends in Medical Education Research. AcadMed, 2004;79(10).

7. Pearson SA, et al. A comparison of practice outcomes of graduates from traditional and non-traditional medical schools in Australia. Med Educ 2002;36(10) 985-991.

8. Khoo HE. Implementation of problem-based learning in Asian medical schools and students' perceptions of their experience. Med Educ 2003;37(5)401-409.

9. Antepohl W, et al. A follow-up of medical graduates of a problem-based learning curriculum. Med Educ 2003;37(2)155-162.

10. O'neill PA, et al. Does a new undergraduate curriculum based on Tomorrow's Doctors prepare house officers better for their first post? A qualitative study of the views of pre-registration house officers using critical incidents. Med Educ 2003;37(12)1100-1108.

11. Prince $\mathrm{KJAH}$, et al. General competencies of problem-based learning (PBL) and non-PBL graduates. Med Educ 2005;39(4)394-401.

12. Schmidt HG, et al. Longterm effects of problem-based learning: a comparison of competencies acquired by graduates of a problem-based and a conventional medical school. Med Educ 2006;40(6)562-567.

13. Srinivasan $\mathrm{M}$, et al. Comparing problem-based learning with case-based learning: effects of a major curricular shift at two institutions. Acad Med 2007;82(1)74-82

14. Antepohl W, Herzig S. Problem-based learning versus lecture-based learning in a course of basic pharmacology: a controlled, randomized study. Med Educ 1999;33(2) 106-113.

15. O'neill PA, et al. Evaluation of an integrated curriculum using problem-based learning in a clinical environment: the Manchester experience. Med Educ 2000;34(3)222-230.

16. Prince $\mathrm{KJAH}$, et al. Does problem-based learning lead to deficiencies in basic science knowledge? An empirical case on anatomy. Med Educ 2003;37(1)15-21.

17. McParland M, et al. The effectiveness of problem-based learning compared to traditional teaching in undergraduate psychiatry. Med Educ 2004;38(8)859

18. Colliver JA. Effectiveness of problem-based learning curricula: research and theory. AcadMed 2000;75(3).

19. Norman GR, Schmidt HG. Effectiveness of problem-based learning curricula: theory, practice and paper darts. Med Educ 2000;34(721).

20. Albanese M. Problem-based learning: why curricula are likely to show little effect on knowledge and clinical skills. Med Educ 2000;34(9)729-738.

21. Cooliver LA. Educational theory and medical education practice: a cautionary note for medical school faculty. AcadMed 2002;77(12)1217-1220.

22. Farrow, R. The effectiveness of PBL: the debate continues. Is meta-analysis helpful? Med Educ 2003;37(12)1131-1132.

23. Moust JHC, Van Berkel HJM, Schmidt HG. Signs of erosion: Reflections on three decades of problem-based learning at Maastricht University. HigherEduc 2005;50.

24. Dolmans DHGM, et al. Problem-based learning: future challenges for educational practice and research. Med Educ 2005;39.

25. Mamede S, Schmidt HG, Norman GR. Innovations in Problem-based Learning: What can we Learn from Recent Studies? Adv in Health Sci Educ;2006.

26. Dauphinee WD.; Wood-Dauphinee S. The need for evidence in medical education: the development of best 
evidence medical education as an opportunity to inform, guide and sustain medical education research. Acad Med 2004;79(10)925-930.

27. Farias PA, et al. Aprendizagem Ativa na Educação em Saúde: Percurso Histórico e Aplicações. RevBrasEducMéd 2015;39(1).

28. Roman AR.Friedlander MR. Revisão integrativa de pesquisa aplicada à enfermagem. CogEnf 1998;03(2).

29. Benefield LE. Implementingevidence-basedpractice in home care. Hom Health Nurs 2003;21(12).

30. Segouin, C. et al. Country report: medical education in France. MedEduc 2007;41(3)295-301.

31. Supe A, Burdick WP. Challenges and issues in medical education in India. Acad Med 2006; 81(12)1-5.

32. Lam T, Wan X, Ip S. Current perspectives on medical education in China. Med Educ 2006;40(10)940-949.

33. Teo A. The current state of medical education in Japan: a system under reform. Med Educ 2007;41(3)302-308.

34. Prideaux D, et al. Country report: Australia. Med Educ 2001;35(5)495-504.

35. Likic R, et al. Analysis and prospects for curricular reform of medical schools in Southeast Europe. Med Educ 2005;39(8)833-840.

36. Almeida MJ Perspectives on South America: the Latin American contribution to the world movement in medical education. Med Educ 2001;35(8) 796-799.

37. Almeida MJ. A educação médica e as atuais propostas de mudança: alguns antecedentes históricos. RevBrasEducMéd 2001;25(2)42-52.

38. Amin Z, et al. Medical education in Southeast Asia: emerging issues, challenges and opportunities. Med Educ $2005 ; 39(8) 829-832$.

39. Hamamoto-Filho PT, et al. Produção Científica sobre Educação Médica no Brasil: Estudo a partir das Publicações da Revista Brasileira de Educação Médica. RevBrasEducMéd 2013;37(4).

40. Cyrino EG, Toralles-Pereira ML. Trabalhando com estratégias de ensino-aprendizado por descoberta na área da saúde: a problematização e a aprendizagem baseada em problemas. CadSaúdPúb 2004;20(3)780-788.

41. Baernstein A, et al. Trends in Study Methods Used in Undergraduate Medical Education Research, 1969-2007. JAMA 2007;298(9).

42. Shea J A, et al. A RIME Perspective on the Quality and Relevance of Current and Future Medical Education Research. Acad Med 2004;79(10).

43. Lira GV. Epistemologia, metodologia e prática de um modelo cartográfico de avaliação curricular em educação médica. Fortaleza; 2010. Doutorado [Tese] - Universidade Federal do Ceará.

44. Thomas G, Pring R. (Orgs). Educação baseada em evidências. Porto Alegre: Artmed, 2007.

45. Guyan GH, et al. User's guide to the medical literature. IX. A method for grading health care recommendations. J Am Med Assoc 1996;275(7)554-558.

46. Fletcher RH, Fletcher SW. Epidemiologia clínica: elementos essenciais. Porto Alegre: Artmed, 2006.

47. Tugwell P, Haynes RB. Avaliando relações de causa e efeito. In: Haynes RB, et al. Epidemiologia clínica: como realizar pesquisa clínica na prática. Porto Alegre: Artmed; 2008. p. 392-425.

48. Torgeson, C.J. Educational research and randomized trials. Med Educ 2002;36(11)1002-1003.

49. Carney PA, et al. Educational epidemiology: applying population-based design and analytic approaches to study medical education. J Am Med Assoc2004;292(9)1044-1050.

50. Celebi N, et al.Problem-based training for medical studentsreduces common prescriptionerrors: a randomisedcontrolledtrial.Med Educ. 2009;43(10)1010-8

51. Albert $\mathrm{M}$ et al. Research in medical education: balancing service and science. Adv in Health SciEduc 2007;12(1)103115.

52. Koh GCH, et al. The effects of problem-based learning during medical school on physician competency: a systematic review. CMA 2008;17(1).

53. Vignochi $C$, et al, Considerações sobre Aprendizagem Baseada em Problemas na Educação em Saúde. Rev. HCPA 2009;29(1).

54. Kinkade S. A Snapshot of the Status of Problem-Based Learning in U. S. Medical Schools, 2003-04. Acad Med. 2005;80(3):300-1

55. Jippes M1, Majoor GD. Influence of national culture on the adoption of integrated and problem-based curricula in Europe. Med Educ. 2008;42(3):279-85.

56. Frambach JM, et al. Rethinking the globalisation of problem-based learning: how culture challenges self-directed learning. Med Educ. 2012;46(8):738-47

57. Fan AP. A snapshot of the status of problem-based learning (PBL) in Chinese medical schools. Med Teach. 2014;36(7):615-20.

\section{CONTRIBUIÇÃO DOS AUTORES}

Ana Neiline Cavalcante contribuiu com o desenho do estudo, coleta, análise, interpretação dos dados e elaboração da versão final para publicação. Geison Vasconcelos Lira e Pedro Gomes Cavalcante Neto contribuíram com o desenho do estudo, a au- 
ditagem do processo de busca, a elaboração da versão final e a revisão crítica para publicação. Roberta Cavalcante Muniz Lira contribuiu com o desenho do estudo e revisões críticas para o encaminhamento à publicação.

\section{CONFLITO DE INTERESSES}

Declarou não haver.

\section{ENDEREÇO PARA CORRESPONDÊNCIA}

Ana Neiline Cavalcante

Av. Comandante Maurocélio Rocha Ponte, 100

Campus Sobral - Sobral

CEP 62042-280 - CE 